\title{
Performance analysis and optimal design for well patterns in anisotropic formations
}

\author{
Liu Yuetian* \\ Key Laboratory of Petroleum Engineering, Ministry of Education, China University of Petroleum, Beijing 102249, China
}

\begin{abstract}
The mechanism of the effects of anisotropic permeability on well patterns and reservoir development are investigated by coordinate transformation, fluid flow analysis, and reservoir development concepts. Anisotropy of permeability has reconstructive effects on well patterns. The originally designed flooding units are broken up, and new pattern units are made up of the wells that belong to different original units. The behavior possesses strong randomness, and leads to a complicated relationship among the injection and production wells, and unpredictable productivity of the formations. To prevent the break-up of well patterns, well lines should be either parallel or perpendicular to the maximum principal direction of the anisotropic permeability (i.e. the fracture direction). To optimize the development effects of anisotropic formations, the latitudinal and longitudinal well spacing of the well network are calculated from the principal values of the anisotropic permeability.
\end{abstract}

Key words: Fractured reservoir, anisotropic permeability, performance analysis, well pattern design

\section{Introduction}

The anisotropy of formation permeability has significant impacts on well patterns and production performance (Chen, 2007; Fokker et al, 2005; Leckenby, et al, 2007; Masihi and King, 2008; Sarma and Aziz, 2006; Tran, et al, 2007). Considerable related research has been done, but most has concerned a specific formation (Chen et al, 2003; Liu et al, 2003; Ma et al, 2000; Salem et al, 2006; Zhou et al, 2002). The conclusions lack universal applicability, and many of them are opposite to one another. This article theoretically analyzes the mechanisms how anisotropic permeability impacts waterflood pattern, and quantitatively investigates the effect on well patterns, and provides commonly-used methods for locating wells in anisotropic formations (especially fractured formations) according to their basic characteristics.

\section{The reconstruction of waterflood patterns}

The phenomenon is analyzed with a five-spot flood pattern in an anisotropic formation as shown in Fig. 1. A rectangular coordinate system is established, in which $x$ and $y$ axes are in the directions of the maximum principal permeability $K_{x}$ and the minimum principal permeability $K_{y}$, respectively. The angle between the maximum principal permeability $K_{x}$ and well lines is $\alpha$.

In order to analyze the effect of permeability anisotropy on well pattern, thereby on production performance, the anisotropic formation is transformed into an equivalent isotropic formation (Collions, 1976; Liu, 2002; 2005; Liu and

\footnotetext{
* Corresponding author. mail: lyt51@163.com

Received December 20, 2007
}

$$
\begin{aligned}
& \text { Zhang, 2004). The following equations are used to calculate } \\
& \text { the fuárłs for } \begin{array}{l}
x^{\prime}=x \sqrt{K / K_{x}} \\
K=\sqrt{K_{x} K_{y}}
\end{array}
\end{aligned}
$$

After coordinate transformation, the anisotropic formation was extended $\sqrt{K / K_{x}}$ and $\sqrt{K / K_{y}}$ times in the $x$ and $y$ directions, respectively. The original anisotropic formation, with principal permeabilities of $K_{x}$ and $K_{y}$, is transformed into an equivalent isotropic formation with a permeability of $K$. The original well pattern in the anisotropic formation (Fig. 1) becomes an equivalent well network in the isotropic

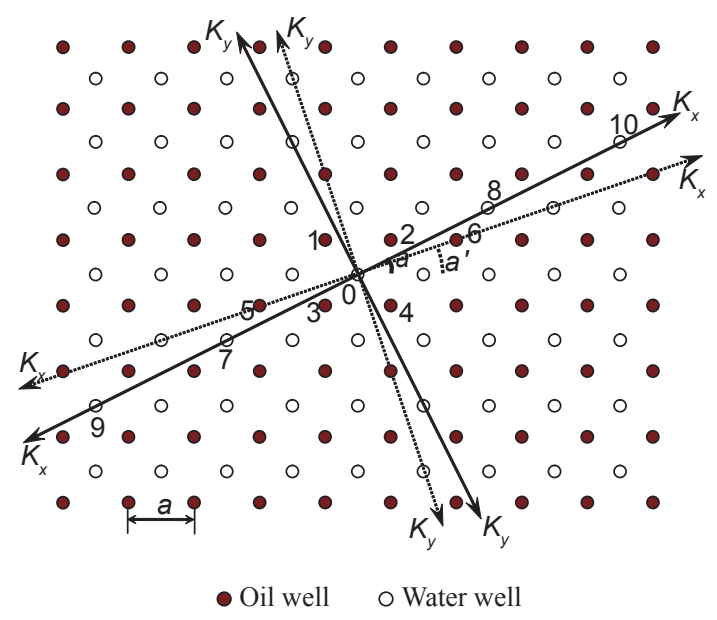

Fig. 1 Five-spot pattern in an anisotropic formation 
formation.

Assuming that $K_{x}>K_{y}$, then $\sqrt{K / K_{x}}<1$ and $\sqrt{K / K_{y}}>1$, the effect of the above coordinate transformation is that the anisotropic formation is compressed to $\left(K_{x} / K_{y}\right)^{1 / 4}$ times in the $x$ direction and elongated to $\left(K_{x} / K_{y}\right)^{1 / 4}$ times in the $y$ direction, therefore the well network takes another form. Assuming that the direction of the maximum principal permeability $K_{x}$ is parallel to the straight line passing through well points 9,7 , 0,8 , and 10 , and $\left(K_{x} / K_{y}\right)^{1 / 4}$ is large enough, the transformed isotropic formation and its well network are shown in Fig. 2.

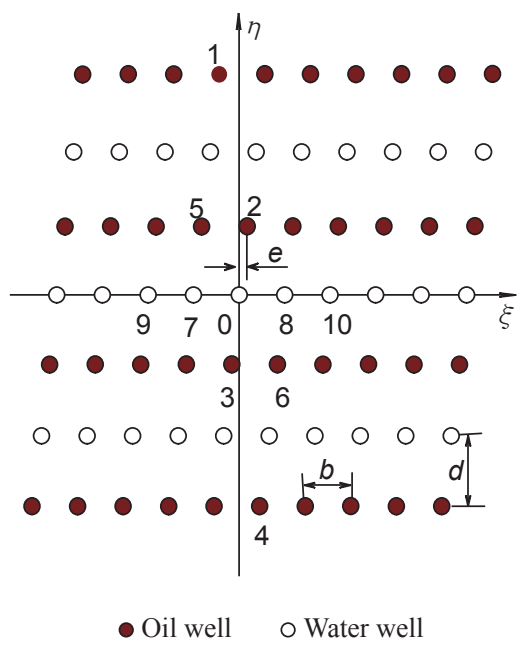

Fig. 2 Line-drive pattern in the equivalent isotropic formation

The five-spot pattern (as shown in Fig. 1) is transformed into the line-drive pattern (as shown in Fig. 2). The flooding unit which consists of wells $0,1,2,3$, and 4 , are broken down. The wells are separated, especially wells 1 and 4 are separated away from well 0 by two well lines. Meanwhile, wells 0,7 , and 8 which are far apart originally become neighboring wells in the same line. The same separation and reconstruction occur for other flooding units. Although the production performance of the line-drive pattern (in Fig. 2 ) is notably different from five-spot pattern in an isotropic formation, it has the equivalent production performance to five-spot pattern in the anisotropic formation as shown in Fig. 1. Even though the production wells 1 and 4 are both located in the flooding unit in which the injection well 0 is located, as shown in Fig. 1, they can hardly be provided with energy by well 0 , and the same results applies to other flooding units. As shown in Fig. 2, because water injection wells are far away from production wells, it is very difficult to transfer the energy from the vicinity of injection wells to the production wells. This always leads to extra high pressure near injection wells, therefore it becomes very difficult to inject water into formation. Meanwhile, extra low pressure appears near production wells and it leads to low productivity. These phenomena appear frequently in the development of fractured reservoirs.

If angle $\quad \alpha$ changes, the transformed well pattern also changes. Assuming that the maximum principal permeability, $K_{x}$ is parallel to the straight line passing through well 5,0 , 6, i.e. $\alpha=\alpha^{\prime}$ (Fig. 1), and that $\left(K_{x} / K_{y}\right)^{1 / 4}$ is large enough, the transformed equivalent isotropic formation and its well pattern are shown in Fig. 3. In the well pattern, production wells and injection wells are located close to each other in the same well lines. So the well pattern is called hybrid line pattern.

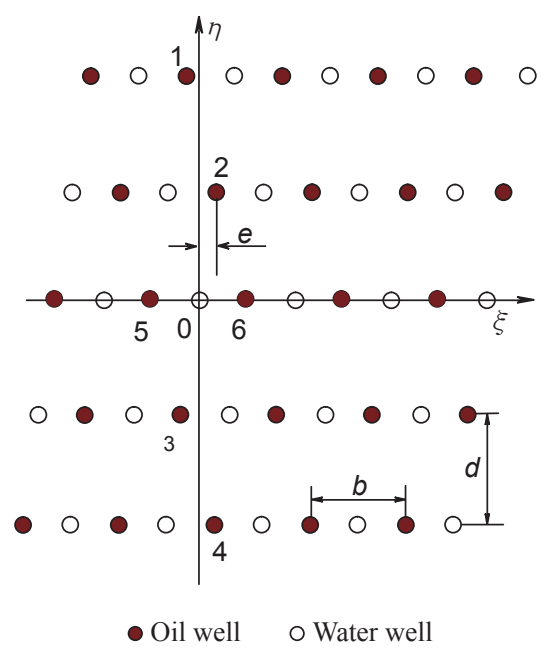

Fig. 3 Hybrid line pattern in the equivalent isotropic formation

The five-spot pattern in anisotropic formations (as shown in Fig. 1) is transformed into the hybrid line pattern in which production wells and injection wells are located close to each other, as shown in Fig. 3. Wells 0, 1, and 4 are separated by two well lines. The injection well 0 and the production wells 5 and 6 , which are far apart originally become neighboring wells in the same line. The hybrid line pattern (in Fig. 3) has the equivalent production performance to the five-spot pattern in anisotropic formations (in Fig. 1). Apparently, water injection performed at any injection well in this well pattern results in fast water breakthrough in the neighboring production wells (Fig. 3), but the flooded-out production wells and the injection well are not located in the same flooding unit originally (Fig. 1). The water cuts of production wells increase quickly. The production rate and recovery of oil stay at a very low level. These phenomena often appear in practical development of reservoirs.

The well pattern in Fig. 1 corresponds to a transformed well pattern for any angle $\alpha$. Theoretically, if $\left(K_{x} / K_{y}\right)^{1 / 4}$ is large enough, the original well pattern will be transformed into a well pattern similar to Fig. 2 when the principal permeability direction is parallel to a straight line passing through any two oil wells, and the original well pattern will be transformed into a well pattern similar to Fig. 3 when the principal permeability direction is parallel to a straight line passing through any one water well and any one oil well. In other cases, original well pattern will be transformed into an irregular pattern between the above two patterns.

Geometrical parameters (such as $b, d, e$ in Figs. 2 and 3 ) of the transformed well patterns are determined by $K_{x}, K_{v}$, 
and $\quad \alpha$. However, the change of transformed well patterns along with $K_{x}, K_{y}$, and $\alpha$ is complicated. The larger the value of $\left(K_{x} / K_{y}\right)^{1 / 4}$ is, the more sensitively the change depends on $\alpha$, and even a slight change of $\alpha$ will result in a completely different transformed well pattern.

The mechanisms of the above change remain true for any type of well patterns in anisotropic formations. The permeability anisotropy in formations has reconstructive effects on well patterns.

\section{Fluid flow and production performance of well patterns}

The transformed line drive pattern (in Fig. 2) and the transformed hybrid line pattern (Fig. 3) are equivalent to the well pattern in Fig. 1 when $a=18.43^{\circ}$ and $\alpha=26.57^{\circ}$, respectively. The geometrical parameters $b, d$, and $e$ in Figs. 2 and 3 are calculated from well spacing $a$, angle $\alpha$, and by Eq. (1). Table 1 presents the calculated values. Supposing that $K=\sqrt{K_{x} K_{y}}$ is constant, well spacing $a=300 \sqrt{2} \mathrm{~m}$ and well bore radius $r=0.1 \mathrm{~m}$ in Fig. 1 .

\subsection{Transformed line drive pattern}

Taking the coordinate system $(o, \xi, \eta)$ as given in Fig. 2, the flow rate and location of a single well in any well line are described as follows.

Production well:

Flow rate $-Q$,

Location $[n b+(2 m+1) e,(2 m+1) d]$.

Injection well:

Flow rate $+Q$,

Location $[n b+2 m e, 2 m d]$.

where $m$ and $n$ are integers and $m \in(-\infty,+\infty), n \in(-\infty,+\infty)$; $b$ is well spacing; $d$ is the distance between two well lines.

The pressure field of a well line parallel to axis $\xi$ is (Muskat, 1937):

$$
p(\xi, \eta)=p_{\mathrm{e}}+q \ln \left[\operatorname{ch} \frac{2 \pi\left(\eta-\eta_{0}\right)}{b}+\cos \frac{2 \pi\left(\xi-\xi_{0}\right)}{b}\right]
$$

with

$$
q=\frac{\mu Q}{4 \pi K h}
$$

where $\left(\xi_{0}, \eta_{0}\right)$ is the location of any one well in the well

\begin{tabular}{|c|c|c|c|c|c|}
\hline$\alpha,^{\circ}$ & $\sqrt{k_{x} / k_{y}}$ & $d / b$ & $e / b$ & $\begin{array}{l}\text { ProductikGily index } \\
h\left(p_{\mathrm{w}}-p_{\mathrm{o}}\right) \\
K\end{array}$ & $\begin{array}{c}\text { Sweep efficiency } \\
E\end{array}$ \\
\hline \multirow{10}{*}{18.43} & 2.00 & 0.77 & 0.15 & 0.422 & 0.522 \\
\hline & 4.00 & 0.80 & 0.40 & 0.429 & 0.679 \\
\hline & 6.00 & 0.67 & 0.67 & 0.444 & 0.586 \\
\hline & 8.00 & 0.80 & 0.20 & 0.459 & 0.398 \\
\hline & 10.00 & 1.00 & 0.20 & 0.472 & 0.318 \\
\hline & 12.00 & 1.20 & 0.20 & 0.484 & 0.265 \\
\hline & 14.00 & 1.40 & 0.20 & 0.495 & 0.227 \\
\hline & 16.00 & 1.60 & 0.20 & 0.505 & 0.199 \\
\hline & 18.00 & 1.80 & 0.20 & 0.514 & 0.177 \\
\hline & 20.00 & 2.00 & 0.20 & 0.523 & 0.159 \\
\hline \multirow{10}{*}{26.57} & 2.00 & 0.77 & 0.35 & 0.430 & 0.414 \\
\hline & 4.00 & 0.80 & 0.90 & 0.444 & 0.398 \\
\hline & 6.00 & 0.67 & 1.17 & 0.447 & 0.477 \\
\hline & 8.00 & 0.80 & 0.30 & 0.444 & 0.602 \\
\hline & 10.00 & 1.00 & 0.30 & 0.437 & 0.659 \\
\hline & 12.00 & 1.20 & 0.30 & 0.428 & 0.705 \\
\hline & 14.00 & 1.40 & 0.30 & 0.419 & 0.743 \\
\hline & 16.00 & 1.60 & 0.30 & 0.408 & 0.773 \\
\hline & 18.00 & 1.80 & 0.30 & 0.398 & 0.797 \\
\hline & 20.00 & 2.00 & 0.30 & 0.387 & 0.817 \\
\hline \multirow{11}{*}{0} & 1.00 & 0.50 & 0.50 & 0.425 & 0.720 \\
\hline & 2.00 & 1.00 & 0.50 & 0.409 & 0.806 \\
\hline & 4.00 & 2.00 & 0.50 & 0.359 & 0.890 \\
\hline & 6.00 & 3.00 & 0.50 & 0.315 & 0.926 \\
\hline & 8.00 & 4.00 & 0.50 & 0.278 & 0.945 \\
\hline & 10.00 & 5.00 & 0.50 & 0.248 & 0.956 \\
\hline & 12.00 & 6.00 & 0.50 & 0.223 & 0.963 \\
\hline & 14.00 & 7.00 & 0.50 & 0.203 & 0.968 \\
\hline & 16.00 & 8.00 & 0.50 & 0.185 & 0.972 \\
\hline & 18.00 & 9.00 & 0.50 & 0.171 & 0.975 \\
\hline & 20.00 & 10.0 & 0.50 & 0.158 & 0.978 \\
\hline
\end{tabular}

Table 1 Well pattern transformation parameters and development indexes of anisotropic formations 
line; $\mu$ is fluid viscosity; $h$ is formation thickness; and $p_{\mathrm{e}}$ is a pressure constant.

The total pressure field of the line-drive pattern can be obtained by superposing all line pressure fields:

$$
\begin{aligned}
p(\xi, \eta)= & p_{\mathrm{e}}-q \ln \left[\operatorname{ch} \frac{2 \pi \eta}{b}-\cos \frac{2 \pi \xi}{b}\right]-q \sum_{m=1}^{\infty}(-1)^{m} \ln \left\{\left[\operatorname{ch} \frac{2 \pi(\eta-m d)}{b}\right.\right. \\
& \left.\left.-\cos \frac{2 \pi(\xi-m e)}{b}\right]\left[\operatorname{ch} \frac{2 \pi(\eta+m d)}{b}-\cos \frac{2 \pi(\xi+m e)}{b}\right]\right\}
\end{aligned}
$$

The fluid flow in the vicinity of any well lines is equivalent to other well lines in the line-drive pattern. The flow rate and flow field in the well pattern are described by analyzing the injection well line at $\eta=0$ and the production well line at $\eta=d$.

\subsubsection{Well productivity}

The bottom hole pressure of the production well $(e, d)$ is:

$$
p_{\mathrm{o}}=p\left(e+r_{\mathrm{w}}, d+r_{\mathrm{w}}\right) \approx p(e, d)
$$

where $r_{\mathrm{w}}$ is the well bore radius, and $r_{\mathrm{w}} \ll e, r_{\mathrm{w}} \ll d$. The bottom hole pressure of the injection well $(0,0)$ is:

$$
p_{\mathrm{w}}=p\left(r_{\mathrm{w}}, r_{\mathrm{w}}\right)
$$

After substituting Eq. (3) into Eqs. (4) and (5), the pressure difference between injection well and production well can be expressed as follows:

$$
\begin{aligned}
p_{\mathrm{w}}-p_{\mathrm{o}}= & 4 q \ln \frac{\left[\operatorname{ch} \frac{2 \pi d}{b}-\cos \frac{2 \pi e}{b}\right]\left[\operatorname{ch} \frac{6 \pi d}{b}-\cos \frac{6 \pi e}{b}\right]}{\sqrt{2} \operatorname{sh} \frac{\pi r_{\mathrm{w}}}{b}\left[\operatorname{ch} \frac{4 \pi d}{b}-\cos \frac{4 \pi e}{b}\right]\left[\operatorname{ch} \frac{8 \pi d}{b}-\cos \frac{8 \pi e}{b}\right]^{1 / 2}+} \\
& 2 q \sum_{m=5}^{\infty}(-1)^{m} \ln \frac{\left[\operatorname{ch} \frac{2(m-1) \pi d}{b}-\cos \frac{2(m-1) \pi e}{b}\right]\left[\operatorname{ch} \frac{2(m+1) \pi d}{b}-\cos \frac{2(m+1) \pi e}{b}\right]}{\left[\operatorname{ch} \frac{2 m \pi d}{b}-\cos \frac{2 m \pi e}{b}\right]^{2}}
\end{aligned}
$$

The combination of Eqs. (4) and (5) also gives:

$$
p_{\mathrm{e}}=\frac{p_{\mathrm{w}}+p_{\mathrm{o}}}{2}
$$

Normally $d / b>\frac{1}{4}$, when $m \geq 4, \operatorname{ch} \frac{2 \pi m d}{b} \approx \frac{1}{2} \exp \left(\frac{2 \pi m b}{b}\right) \gg 1$, $\operatorname{ch} \frac{2 \pi m d}{b} \gg\left|\cos \frac{8 \pi e}{b}\right|$, Eq. (6) can be simplified as follows:

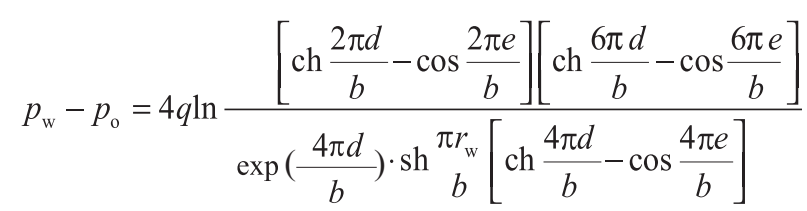

Substituting $q=\frac{\mu Q}{4 \pi K h}$ into Eq. (8) gives the single well productivity:

$Q=\pi K h\left(p_{\mathrm{w}}-p_{\mathrm{o}}\right)\left[\mu \ln \frac{\left[\operatorname{ch} \frac{2 \pi d}{b}-\cos \frac{2 \pi e}{b}\right]\left[\operatorname{ch} \frac{6 \pi d}{b}-\cos \frac{6 \pi e}{b}\right]}{\exp \left(\frac{4 \pi d}{b}\right) \operatorname{sh} \frac{\pi r_{w}}{b}\left[\operatorname{ch} \frac{4 \pi d}{b}-\cos \frac{4 \pi e}{b}\right]}\right]^{-1}$

\subsubsection{Sweep efficiency}

Because of the symmetry of the well pattern, it is necessary only to study the flow in the belt-zone of $-d \leq \eta \leq d$. In general $d / b>\frac{1}{4}$, only the terms with $m \leq 4$ in Eq. (3) have effect on the belt-zone of interest. Eq. (3) then can be written as:

$p(\xi, \eta)=p_{\mathrm{e}}-q \sum_{m=-4}^{+4}(-1)^{m} \ln \left[\operatorname{ch} \frac{2 \pi(\eta-m d)}{b}-\cos \frac{2 \pi(\xi-m e)}{b}\right], \quad 0 \leq \eta \leq d$

The flow velocities in the $\xi$ and $\eta$ directions at any point in the formation can be derived from Eq. (10):

$$
\begin{aligned}
& v_{\xi}=-\frac{K}{\mu} \frac{\partial p}{\partial \xi} \\
& v_{\eta}=-\frac{K}{\mu} \frac{\partial p}{\partial \eta}
\end{aligned}
$$

The water breakthrough time $\left(T_{\mathrm{b}}\right)$ of the well pattern is defined as the traveling time of injected water from the injection well $(0,0)$ to the production well $(e, d)$ along the main stream line $\left(s_{\mathrm{m}}\right)$ :

$$
T_{\mathrm{b}}=\int_{(0,0)}^{(e, d)}\left(v_{\xi}^{2}+v_{\eta}^{2}\right)^{-1 / 2} \mathrm{~d} s_{\mathrm{m}}
$$

where $T_{\mathrm{b}}$ can be calculated using numerical integral.

Because the injection well density in this pattern is 
$1 /(2 b d)$, the sweep efficiency at the water breakthrough time is:

$$
E=T_{\mathrm{b}} \cdot Q /(2 \phi h b d)
$$

The productivity index $\left(\frac{\mu Q}{K h\left(p_{\mathrm{w}}-p_{\mathrm{o}}\right)}\right)$ and sweep efficiency $(E)$ of the transformed line drive pattern can be calculated from Eqs. (9) and (12), as shown in Table 1.

\subsection{Transformed hybrid line pattern}

As shown in Fig. 3, the flow rate and location of a single well in each well line are described as follows.

Production well:

Flow rate $-Q$,
Location $[n b+(2 m+1) e,(2 m+1) d]$ or $\left[n b+\frac{1}{2} \mathrm{~b}+2 m e, 2 m d\right]$.

Injection well:

Flow rate $+Q$,
Location $[n b+2 m e, 2 m d]$ or $\left[n b+\frac{1}{2} \mathrm{~b}+(2 m+1) e,(2 m+1) d\right]$.

where $m$ and $n$ are integers and $m \in(-\infty,+\infty), n \in(-\infty,+\infty)$.

The pressure distribution of the whole well network is:

$$
\begin{aligned}
p(\xi, \eta)= & q \ln \left[\left(\operatorname{ch} \frac{2 \pi \eta}{b}+\cos \frac{2 \pi \xi}{b}\right) /\left(\operatorname{ch} \frac{2 \pi \eta}{b}-\cos \frac{2 \pi \xi}{b}\right)\right]- \\
& q \sum_{m=1}^{\infty}(-1)^{m} \ln \left\{\left[\operatorname{ch} \frac{2 \pi(\eta-m d) \pi}{b}-\cos \frac{2 \pi(\xi-m e)}{b}\right]\left[\operatorname{ch} \frac{2 \pi(\eta+m d)}{b}-\cos \frac{2 \pi(\xi+m e)}{b}\right]\right\} \\
& +q \sum_{m=1}^{\infty}(-1)^{m} \ln \left\{\left[\operatorname{ch} \frac{2 \pi(\eta-m d)}{b}+\cos \frac{2 \pi(\xi-m e)}{b}\right]\left[\operatorname{ch} \frac{2 \pi(\eta+m d)}{b}+\cos \frac{2 \pi(\xi+m e)}{b}\right]\right\}
\end{aligned}
$$

Using the same steps mentioned in section 3.1, the productivity index $\left(\frac{\mu Q}{K h\left(p_{\mathrm{w}}-p_{\mathrm{o}}\right)}\right)$ and sweep efficiency $(E)$ of the transformed hybrid line pattern can be calculated from Eq. (13), as shown in Table1.

\subsection{Analysis}

Table 1 shows that the well productivities and sweep efficiencies corresponding to $\quad \alpha=18.43^{\circ}$ are completely different from those corresponding to $\alpha=26.57^{\circ}$, and they vary complicatedly along with the change of $\sqrt{K_{x} / K_{y}}$.

Geometrical parameters $(d / b$ and $e / b)$ of the transformed well pattern also change irregularly. This indicates that both the change in the value and direction of maximum principal permeability can result in significant variation in well pattern and production performance of anisotropic formations.

\section{Method for locating wells in anisotropic formations}

\subsection{Commonly-used method for locating wells}

It should be noted that, if the straight line passing through any two wells in the same flooding unit is parallel to the maximum principal permeability, the flooding unit will not be broken up when the anisotropic formation is transformed into an equivalent isotropic formation. Only the shape of flooding units changes, and the parameters are determined by the strength of anisotropy only.

When the direction of injection well lines is parallel to the principal direction of maximum permeability (i.e. fracture direction) ( $\alpha=0^{\circ}$, as shown in Fig. 1), a rectangular pattern in anisotropic formations can be transformed into an equivalent rectangular pattern in isotropic formations, as shown in Figs. 4 and 5 .

Thus, well-pattern arrangement in anisotropic formations can be described as follows:

1) The direction of well lines should be parallel or perpendicular to the direction of maximum principal permeability. The well line is the line passing through any two well points in the same flooding unit, and the direction of maximum principal permeability is the fracture direction or palaeo-current direction in the formation sediments.

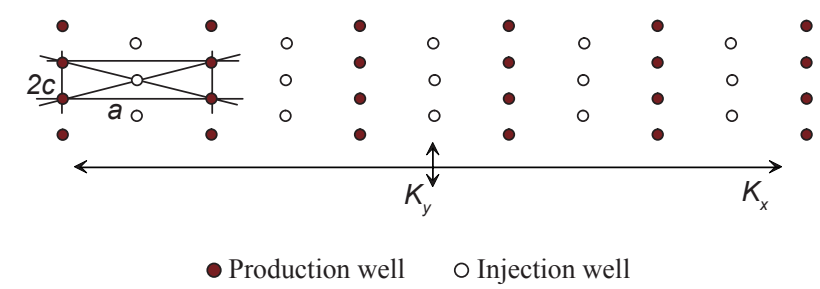

Fig. 4 Five-spot rectangular pattern in the anisotropic formation

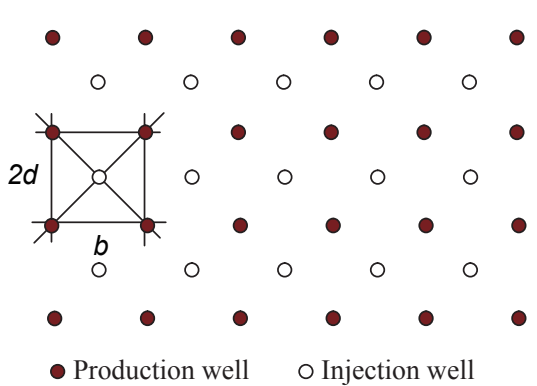

Fig. 5 Five-spot rectangular pattern in the equivalent isotropic formation 
2) The geometrical parameters for well pattern design of anisotropic formations are given by:

$$
\left\{\begin{array}{l}
a=b \sqrt{K_{x} / K} \\
c=d \sqrt{K_{y} / K} \\
K=\sqrt{K_{x} K_{y}}
\end{array}\right.
$$

where $a$ and $c$ are the latitudinal and longitudinal well spacings in anisotropic formations; $b$ and $d$ are the latitudinal and longitudinal well spacings in the equivalent isotropic formations; $K_{x}$ and $K_{y}$ are the maximum and minimum principal values of the anisotropic permeability, respectively.

\subsection{Design of 5-point rectangular pattern}

As shown in Fig. 4, injection well is aligned on the diagonal of the rectangular pattern, parallel to the direction of the maximum principal permeability (fracture direction), i.e. $\alpha=0^{\circ}$, which is a special case of Fig. 2, and

$$
\left\{\begin{array}{l}
b=\frac{a}{\sqrt{K_{x} / K}} \\
d=c \sqrt{K_{x} / K} \\
e / b \equiv 0.5
\end{array}\right.
$$

Substituting Eq. (15) into Eq. (9) gives the productivity of the rectangular patterns:

$$
Q=\frac{\pi K h\left(p_{\mathrm{w}}-p_{\mathrm{o}}\right)}{\mu\left[\ln \left(2 \operatorname{ch}^{2} \frac{\pi d}{b} \operatorname{ch}^{2} \frac{3 \pi d}{b}\right)-\ln \left(\exp \left(\frac{4 \pi d}{b}\right) \operatorname{sh} \frac{\pi r_{\mathrm{w}}}{b} \operatorname{sh}^{2} \frac{2 \pi d}{b}\right)\right]}
$$

The sweep efficiency of rectangular patterns can be calculated from Eqs. (10)-(12) and it also can be calculated from the formulas given by Prats (1956) due to the regular shape of the patterns:

$$
\begin{aligned}
& E=\frac{\pi}{2} \frac{G\left[\left(g-g^{\prime}\right)^{2}\right]}{G\left(g^{\prime}\right) G(g)} \\
& \frac{d}{b}=\frac{1}{2} \frac{G\left(g^{\prime}\right)}{G(g)}
\end{aligned}
$$

where $G(g)$ ad $G\left(g^{\prime}\right)$ are functions of the complete elliptical integral of the first kind with its modulus $g, g^{2}+g^{\prime 2}=1$.

When designing a well pattern, the productivity and sweep efficiency under different geometrical parameters $b$ and $d$ should first be calculated from Eqs. (16) and (17), and the productivity and sweep efficiency are optimized by choosing the appropriate geometrical parameters $b$ and $d$. Then the geometrical parameters $a$ and $c$ of well pattern in anisotropic formations are determined by Eq. (14). As an example, calculated productivity index and sweep efficiency of the case with $b \times d=a \times c=0.09 \mathrm{~km}^{2}$ are shown in Table 1 $\left(\alpha=0^{\circ}\right)$.

\section{Conclusions}

1) Permeability anisotropy has reconstructive effects on water flood patterns.

2) To prevent the well pattern break-up, well lines should be either parallel or perpendicular to the direction of the maximum principal permeability in anisotropic formations.

3) The production performance of anisotropic formations can be optimized through adjusting the latitudinal and longitudinal well spacings, which are determined by the principal values of the anisotropic permeability.

\section{References}

Chen G L, Liang C X, Liu Z L, et al. Optimization of the flooding pattern in reservoirs with obvious directions of fracture and sand body. Petroleum Exploration and Development. 2003. 31(4): 112-115 (in Chinese)

Chen Z X. Homogenization and simulation for compositional flow in naturally fractured reservoirs. Journal of Mathematical Analysis and Applications. 2007. 326(1): 12-32

Collions R E. Flow of fluids through porous materials. Tulsa. The Petroleum Publishing Company. 1976. 74-76

Fokker P A, Verga F and Egberts P J P. New semianalytic technique to determine horizontal well productivity index in fractured reservoirs. SPE Reservoir Evaluation \& Engineering. 2005. 8(2): 123-131

Leckenby R J, Lonergan L, Rogers S F, et al. Study of fracture-induced anisotropy from discrete fracture network simulation of well test responses. Geological Society, London, Special Publications. 2007. 270: $117-137$

Liu Y T. Analysis on seepage flow and productivity of a horizontal well in an anisotropic reservoir. Journal of the University of Petroleum, China. 2002. 26(4): 41-47 (in Chinese)

Liu Y T. Well location in water-flooding anisotropic reservoirs. Petroleum Exploration and Development. 2005. 32(1): 101-104 (in Chinese)

Liu Y T and Zhang J C. Stable permeating flow and productivity analysis for anisotropic reservoir in horizontal well networks. Petroleum Exploration and Development. 2004. 31(1): 94-96 (in Chinese)

Liu Z L, Wei Z S, Chen W L, et al. Optimal flooding patterns in fractured low-permeability sandstone reservoir. Petroleum Exploration and Development. 2003. 30(4): 85-88 (in Chinese)

Ma L W, Guan Y D and Han P R. Practice and recognition on pattern adjustment in fractural low permeable sandstone oilfield. Petroleum Geology \& Oilfield Development in Daqing. 2000. 19(3): 11-14 (in Chinese)

Masihi M and King P R. Connectivity prediction in fractured reservoirs with variable fracture size: Analysis and validation. SPE Journal. 2008. 13(1): 88-98

Muskat M. The flow of homogeneous fluids through porous media. New York and London: McGraw-Hill Book Company Inc. 1937. 523-530

Prats M. The breakthrough sweep efficiency of the staggered line drive. Petroleum Transactions, AIME. 1956. 207: 26-28

Salem S E, Al-Deeb M, Abdou M, et al. Practical flow-simulation 
method for a naturally fractured reservoir: A field study. SPE Reservoir Evaluation \& Engineering. 2006. 9(2): 173-185

Sarma P and Aziz K. New transfer functions for simulation of naturally fractured reservoirs with dual-porosity models. SPE Journal. 2006. 11(3): 328-340

Tran N H, Chen Z and Rahman S S. Practical application of hybrid modelling to naturally fractured reservoirs. Petroleum Science and Technology. 2007. 25(10): 1263-1277

Zhou Z J, Song H C, Meng L B, et al. Numerical simulation of wellpattern optimization in low permeability fractured reservoir: An example of Liangjing Oilfield. Xinjiang Petroleum Geology. 2002. 23(3): 228-230 (in Chinese)

(Edited by Sun Yanhua) 